\title{
Circulating ApoE Level is Independently Associated with Urinary Albumin Excretion in Type 2 Diabetic Patients
}

\author{
Yan Ling ${ }^{1}$, Xiaomu $\mathrm{Li}^{1}$, Qian $\mathrm{Gu}^{2}$ and Xin $\mathrm{Gao}^{1}$
}

\begin{abstract}
Objective Evidence from many studies suggests that dyslipidemia has a pathogenetic role in the development and progression of diabetic nephropathy. The objective of this study was to evaluate the relationships between serum lipid parameters and urinary albumin excretion in type 2 diabetic patients from Shanghai, China.

Methods A total of 1094 type 2 diabetic patients were recruited. The urinary albumin to creatinine ratio (ACR), serum total cholesterol, triglyceride, high density lipoprotein cholesterol (HDL-C), apolipoprotein A I (ApoA I), apolipoprotein B (ApoB), apolipoprotein E (ApoE) and lipoprotein (a) [Lp(a)] were measured and low density lipoprotein cholesterol (LDL-C) was calculated according to the Friedewald formula. An ACR of $\geq 30 \mu \mathrm{g} / \mathrm{mg}$ was defined as albuminuria, and $<30 \mu \mathrm{g} / \mathrm{mg}$ as normoalbuminuria.

Results The levels of ApoE and triglyceride were significantly higher in albuminuric group compared with normoalbuminuric group $(\mathrm{p}<0.001$ and $\mathrm{p}=0.003$, respectively). Differences of ACR among the groups based on the tertile of ApoE and the tertile of triglyceride were significant (both $\mathrm{p}<0.001$ ). In a multiple linear regression model, ApoE was independently associated with ACR $(\beta=0.003$, 95\% CI $0.002-0.005, \mathrm{p}<0.001)$.

Conclusion Serum ApoE level was independently associated with urinary albumin excretion in type 2 diabetic patients from Shanghai, China.
\end{abstract}

Key words: type 2 diabetes, apolipoprotein E, urinary albumin, lipids

(Intern Med 50: 2961-2966, 2011)

(DOI: 10.2169/internalmedicine.50.6216)

\section{Introduction}

Diabetic nephropathy is a major cause of end-stage renal disease and an important risk factor for atherosclerotic cardiovascular disease. Evidence from many studies suggests that dyslipidemia has a pathogenetic role in the development and progression of diabetic nephropathy (1-4). Lipoprotein abnormalities associated with diabetic nephropathy include increased plasma levels of very low density lipoprotein (VLDL), intermediate-density lipoprotein (IDL), and LDL and decreased concentrations of HDL (3). Diabetes is also associated with alterations in the amounts of several classes of apolipoproteins, including apolipoprotein A I (ApoA I), apolipoprotein AII (ApoA II), apolipoprotein B (ApoB), apolipoprotein C I(ApoC I) and apolipoprotein C III (ApoC
III) $(4,5)$, which can affect the development of nephropathy in patients with diabetes. The mechanisms by which lipids induce renal injury in diabetic patients include excess production of reactive oxygen species, activation of TGF- $\beta$ pathway $(6,7)$, disruption of endothelial cell glycocalyx $(8,9)$ and monocyte transmigration (10). Diabetic nephropathy is characterized by a progressive increase of urinary albumin excretion. The association of dyslipidemia with albuminuria in Chinese type 2 diabetic patients has only been examined in Taiwanese. Two studies found that apoB, triglyceride and lipoprotein (a) $[\mathrm{Lp}(\mathrm{a})]$ were associated with albuminuria in type 2 diabetic patients from Taiwan $(11,12)$. The association of ApoE with albuminuria was not investigated in either of these studies. The objective of the present study was to evaluate the relationships between urinary albumin excretion and the serum lipid pa-

${ }^{1}$ Department of Endocrinology and Metabolism, Zhongshan Hospital, Fudan University, PR China and ${ }^{2}$ Department of Geriatrics, Zhongshan Hospital, Fudan University, PR China

Received for publication July 20, 2011; Accepted for publication September 11, 2011

Correspondence to Dr. Xin Gao, gao.xin@zs-hospital.sh.cn 
rameters, including total cholesterol, triglycerides, HDL-C, LDL-C, ApoA I, ApoB, ApoE and Lp(a) in type 2 diabetic patients from Shanghai, China.

\section{Materials and Methods}

\section{Subjects}

During January 2007 to December 2008, a total of 1094 type 2 diabetic patients were recruited from the Endocrinology and Metabolism Department of Zhongshan Hospital in Shanghai, which is affiliated to Fudan University. All participants were of Southern Han Chinese ancestry and resided in the Shanghai metropolitan area, met the 1999 WHO criteria for diabetes (13), had been diagnosed after the age of 29 years, and were treated with oral hypoglycemic agents and/ or insulin. Exclusion criteria were diabetes other than type 2 diabetes, known nondiabetic kidney disease, renal transplant or on dialysis, renal vascular disease, obstructive uropathy, infection, fever, congestive heart failure, and pregnancy.

Written informed consent was obtained from all participants and the study was approved by the ethnic committee of Zhongshan Hospital, Fudan University, Shanghai, China.

\section{Clinical measurements}

\section{ACR and estimated glomerular filtration rate (eGFR)}

Urinary specimens were collected in the morning after the subjects fasted for at least 12 hours. First-void and midstream urine was collected. The concentration of urinary albumin and urinary creatinine were measured by Hitachi 7600 biochemistry autoanalyzer [albumin: immunoturbidimetric assay, DiaSys Diagnostic Systems (Shanghai) Co., Ltd; creatinine: enzymatic assay, Roche Diagnostica, Basel, Switzerland]. ACR was calculated by dividing the urinary albumin concentration in micrograms by the urinary creatinine concentration in milligrams. An ACR $\geq 30 \mu \mathrm{g} / \mathrm{mg}$ was defined as albuminuria, and $<30 \mu \mathrm{g} / \mathrm{mg}$ as normoalbuminuria. eGFR ( $\mathrm{mL} / \mathrm{min})$ was calculated from the Cockcroft-Gault formula as: [(140-age in years) * body weight in $\mathrm{kg}] /(72 *$ serum creatinine in $\mathrm{mg} / \mathrm{dL})(14)$. For women, the calculated values were multiplied by 0.85 (14).

\section{Lipid parameters}

Blood samples were collected in tubes without anticoagulant in the morning after the subjects fasted for at least 12 hours. The samples were allowed to clot at room temperature, and serum was obtained by centrifugation at 3,000 rpm for 15 minutes. Lipid profile was determined with Hitachi 7600 biochemistry autoanalyzer. Triglyceride, total cholesterol and HDL-C were measured with enzymatic methods (triglyceride, lipase-GPO-PAP method, Shanghai Kehuadongling Diagnostics Co., Ltd.; total cholesterol, CHODPAP method, Shanghai Kehuadongling Diagnostics Co., Ltd.; HDL-C, PEG-modified enzymatic assay, Kyowa Medex Co., Ltd., Tokyo). LDL-C was calculated according to the Friedewald formula (15). ApoA-I, ApoE, ApoB, and $\mathrm{Lp}$ (a) were determined by immunoturbidimetric assays (ApoA-I and Apo B, DiaSys Diagnostic Systems Co., Ltd.; Apo E and Lp(a), Nittobo Boseki Co., Ltd., Tokyo).

\section{Potential confounders}

Age, sex, body mass index (BMI), duration of diabetes, history of hypertension, duration of hypertension, glycosylated hemoglobin (HbA1c), systolic blood pressure (SBP), diastolic blood pressure (DBP), serum creatinine, the use of statin/fibrate and the use of angiotensin-converting enzyme inhibitor (ACEI)/angiotensin receptor blocker (ARB) were treated as potential confounders.

Blood pressure was measured on the right arm after 20 min rest in a sitting position with a standard mercury sphygmomanometer. Body height in centimeters and body weight in kilograms $(\mathrm{kg})$ were measured with light clothes and bare feet, and BMI in $\mathrm{kg} / \mathrm{m}^{2}$ was calculated. Serum creatinine and plasma glucose levels were measured by Hitachi 7600 biochemistry autoanalyzer (creatinine: enzymatic assay, Roche Diagnostica, Basel, Switzerland; glucose: glucose oxidase method, Shanghai Kehuadongling Diagnostics Co., Ltd.). HbA1c was measured by high performance liquid chromatography using the Bio-Rad Variant II analyzer (Bio-Rad Laboratories, Hercules, CA, USA).

\section{Statistical analyses}

Continuous variables were expressed as the mean \pm standard error of mean (SEM) or median (interquartile range), and categorical variables were expressed as percentages. Comparisons between groups were performed with $\mathrm{T}$ test or one-way ANOVA and Chi-squared test for normally distributed continuous and categorical variables, respectively. The post hoc method used in one-way ANOVA was Fisher's least significant difference (LSD). The stepwise multiple linear regression model was used to assess independent relationships between lipid parameters and ACR. Non-normally distributed values were natural log-transformed before analysis if necessary. Two-sided $\mathrm{p}<0.05$ was considered statistically significant. All statistical analyses were performed with SPSS software version 17.0.

\section{Results}

Among the 1094 type 2 diabetic patients, 744 had normoalbuminuria and 350 had albuminuria. Comparisons of the baseline characteristics between the normoalbuminuric and albuminuric groups are shown in Table 1. Subjects with albuminuria were older, had a higher proportion of hypertension, longer duration of diabetes and hypertension, higher SBP, higher BMI, higher triglyceride and ApoE levels, higher serum creatinine, and lower eGFR. More patients in the albuminuric group were receiving treatment with insulin and ACEI or ARBs.

The levels of ApoE and triglyceride were significantly higher in the albuminuric group compared with the normoal- 
Table 1. Baseline Characteristics of Study Subjects

\begin{tabular}{|c|c|c|c|c|}
\hline Variables & $\begin{array}{c}\text { Total } \\
(n=1094)\end{array}$ & $\begin{array}{l}\text { Normoalbuminuria } \\
\qquad(\mathrm{n}=744)\end{array}$ & $\begin{array}{l}\text { Albuminuria } \\
\qquad(n=350)\end{array}$ & p value* \\
\hline Age (years) & $59.9 \pm 0.39$ & $58.3 \pm 0.47$ & $62.9 \pm 0.71$ & $<0.001$ \\
\hline Gender (\% men) & 45.7 & 48.0 & 41.7 & 0.05 \\
\hline BMI $\left(\mathrm{kg} / \mathrm{m}^{2}\right)$ & $24.2 \pm 0.11$ & $24.0 \pm 0.13$ & $24.9 \pm 0.22$ & $<0.001$ \\
\hline FPG $(\mathrm{mmol} / \mathrm{L})$ & $7.81 \pm 0.22$ & $7.76 \pm 0.31$ & $7.95 \pm 0.24$ & 0.71 \\
\hline HbAlc (\%) & $9.40 \pm 0.10$ & $9.31 \pm 0.08$ & $9.48 \pm 0.12$ & 0.26 \\
\hline Duration of diabetes (years) & $7.73 \pm 0.25$ & $6.88 \pm 0.30$ & $8.02 \pm 0.49$ & $<0.001$ \\
\hline SBP (mmHg) & $132 \pm 0.48$ & $130 \pm 0.56$ & $137 \pm 0.95$ & $<0.001$ \\
\hline $\mathrm{DBP}(\mathrm{mmHg})$ & $80.2 \pm 0.27$ & $80.0 \pm 0.33$ & $81.1 \pm 0.51$ & 0.08 \\
\hline Hypertension (\%) & 55.8 & 49.3 & 68.7 & $<0.001$ \\
\hline Duration of hypertension (years) & $6.66 \pm 0.36$ & $5.61 \pm 0.41$ & $9.08 \pm 0.72$ & $<0.001$ \\
\hline $\mathrm{ACR}^{\#}(\mathrm{ug} / \mathrm{mg})$ & $13.3(6.03-47.5)$ & $7.95(4.40-14.0)$ & $105(50.5-322)$ & $<0.001$ \\
\hline Serum creatinine (umol/L) & $71.9 \pm 1.34$ & $66.3 \pm 1.05$ & $82.9 \pm 3.70$ & $<0.001$ \\
\hline eGFR (mL/min) & $93.6 \pm 1.10$ & $97.4 \pm 1.28$ & $87.3 \pm 2.30$ & $<0.001$ \\
\hline Total cholesterol (mmol/L) & $4.56 \pm 0.05$ & $4.53 \pm 0.06$ & $4.61 \pm 0.11$ & 0.46 \\
\hline Triglyceride $^{\#}(\mathrm{mmol} / \mathrm{L})$ & $1.47(1.00-2.26)$ & $1.38(1.00-2.07)$ & $1.75(1.11-2.68)$ & $<0.001$ \\
\hline HDL-C (mmol/L) & $1.22 \pm 0.01$ & $1.23 \pm 0.01$ & $1.19 \pm 0.03$ & 0.15 \\
\hline LDL-C (mmol/L) & $2.51 \pm 0.03$ & $2.51 \pm 0.03$ & $2.48 \pm 0.07$ & 0.67 \\
\hline ApoA I $\quad(g / L)$ & $1.03 \pm 0.01$ & $1.03 \pm 0.01$ & $1.04 \pm 0.02$ & 0.76 \\
\hline ApoB (g/L) & $0.90 \pm 0.01$ & $0.90 \pm 0.01$ & $0.91 \pm 0.02$ & 0.57 \\
\hline ApoE (mg/L) & $53.5 \pm 0.94$ & $51.2 \pm 0.95$ & $58.9 \pm 2.34$ & 0.003 \\
\hline $\mathrm{Lp}(\mathrm{a})(\mathrm{mg} / \mathrm{L})$ & $184 \pm 5.79$ & $176 \pm 6.68$ & $199 \pm 11.9$ & 0.10 \\
\hline Insulin $(\%)$ & 28.5 & 26.5 & 32.8 & 0.03 \\
\hline ACEI/ARB (\%) & 21.1 & 17.9 & 27.5 & 0.002 \\
\hline Statins/fibrates (\%) & 50.2 & 47.6 & 53.0 & 0.09 \\
\hline
\end{tabular}

Continuous data are expressed as means \pm SEM and median (interquartile range)

\#Variables were natural log-transformed before statistical analysis

${ }^{*} \mathrm{p}$ value for comparison between subjects without albuminuria and subjects with albuminuria.

ACEI: angiotensin-converting enzyme inhibitor; ACR: albumin-to-creatinine ratio; ARB: angiotensin receptor blocker; ApoA I : apolipoprotein A I ; ApoB: apolipoprotein B; ApoE: apolipoprotein E; BMI: body mass index; DBP: diastolic blood pressure; eGFR: estimated glomerular filtration rate; FPG: fasting plasma glucose; HbA1c: glycosylated hemoglobin; HDL-C: high density lipoprotein cholesterol; LDL-C: low density lipoprotein cholesterol; Lp(a): lipoprotein (a); SBP: systolic blood pressure.
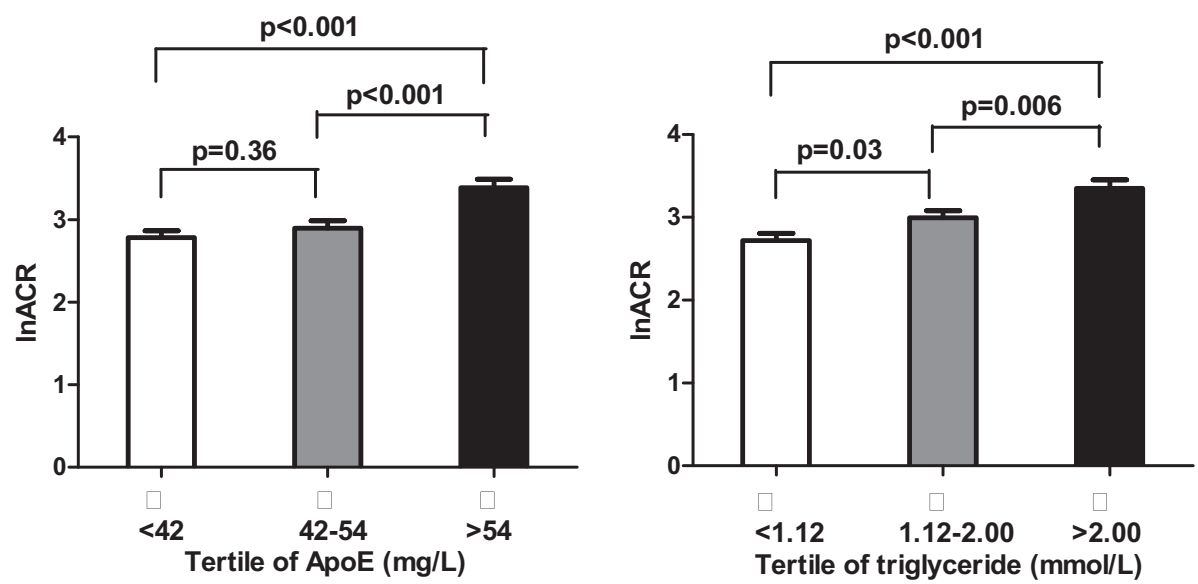

Figure 1. Urinary albumin to creatinine ratio by tertiles of serum apolipoprotein $\mathbf{E}$ and triglyceride levels. InACR: natural log-transformed albumin to creatinine ratio

buminuric group (Table 1). We classified the study subjects into three groups based on the tertiles of ApoE level $(<42$, $42-54,>54 \mathrm{mg} / \mathrm{L})$ or triglyceride level $(<1.12,1.12-2.00$, $>2.00 \mathrm{mmol} / \mathrm{L}$ ). Differences of ACR among the groups based on the tertile of ApoE level were significant $(\mathrm{p}<$
0.001), and the ACR level increased across the tertile groups (Fig. 1). The third ApoE tertile group had a significantly higher ACR compared with the first and the second groups (both $\mathrm{p}$ values $<0.001$ ). The differences of ACR among the groups based on the tertile of triglyceride level were also 
Table 2. Multiple Linear Regression Analysis of Relationships between Selected Variables and LnACR

\begin{tabular}{lccc}
\hline \multicolumn{1}{c}{ Variables } & $\beta$ & $95 \%$ Confidence interval & $\mathrm{p}$ value \\
\hline Duration of diabetes & 0.016 & $0.008-0.024$ & $<0.001$ \\
HbAlc & 0.034 & $0.010-0.057$ & 0.005 \\
BMI & 0.041 & $0.025-0.058$ & $<0.001$ \\
SBP & 0.009 & $0.005-0.012$ & $<0.001$ \\
ApoE & 0.003 & $0.002-0.005$ & $<0.001$ \\
eGFR & -0.006 & $-0.008--0.004$ & $<0.001$ \\
\hline
\end{tabular}

In addition to the significant variables shown in the table, the multiple linear regression model also included sex, age, hypertension duration, diastolic blood pressure, total cholesterol, triglyceride, low density lipoprotein cholesterol, high density lipoprotein cholesterol, apolipoprotein A I , apolipoprotein B, lipoprotein (a), use of insulin, use of angiotensin-converting enzyme inhibitor or angiotensin receptor blocker and use of statins or fibrates.

ApoE: apolipoprotein E; BMI: body mass index; eGFR: estimated glomerular filtration rate; HbAlc: glycosylated hemoglobin; lnACR: natural log-transformed albumin to creatinine ratio; SBP: systolic blood pressure.

significant $(\mathrm{p}<0.001)$, and the ACR level increased across the tertile groups (Fig. 1). The third triglyceride tertile group had significantly higher ACR compared with the first and the second groups $(\mathrm{p}<0.001$ and $\mathrm{p}=0.006$, respectively), and the second group also had a significantly higher ACR compared with the first group $(\mathrm{p}=0.03)$.

We next investigated the relationships between lipid parameters and ACR in a multiple linear regression model which included sex, age, BMI, duration of diabetes, duration of hypertension, SBP, DBP, HbA1c, total cholesterol, triglyceride, LDL-C, HDL-C, ApoA1, ApoB, ApoE, Lp(a), eGFR, use of insulin, use of ACEI or ARBs and use of statins or fibrates as variables. We found that $\mathrm{ApoE}$ was independently associated with ACR $(\beta=0.003$, 95\%CI 0.002 $0.005, \mathrm{p}<0.001$ ) (Table 2). Other predictors of ACR were the duration of diabetes, HbA1c, SBP, BMI and eGFR (Table 2).

\section{Discussion}

The present study demonstrated that serum ApoE level was independently associated with urinary albumin excretion as determined by ACR in type 2 diabetic patients from Shanghai, China. ACR increased gradually with increasing tertiles of ApoE level as shown in one-way ANOVA analysis. In the multiple linear regression analysis, after adjusting for common risk factors of diabetic nephropathy and including other lipid parameters and medication use in the model, the serum ApoE level was significantly and positively associated with ACR in the study population.

$\mathrm{ApoE}$ is a class of apolipoprotein, and is an important component of chylomicron, IDL and VLDL (16). It is essential for the normal catabolism of triglyceride-rich lipoproteins (16). ApoE was initially recognized for its importance in cardiovascular disease (17) and Alzheimer's disease (18). There are also many studies indicating that ApoE has a major role in the pathogenesis and progression of a variety of renal diseases. ApoE gene mutations and elevated ApoE concentrations contribute to the development of lipo- protein glomerulopathy (19). Lipoprotein glomerulopathy is a unique lipidosis characterized by peculiar histopathologic characteristics of lipoprotein thrombi, protinuria and an abnormal plasma lipoprotein profile resembling type III hyperlipoproteinemia, with a marked increase in serum ApoE concentrations (19). ApoE is also associated with the development and progression of other glomerulopathies $(20,21)$.

The present study found that ApoE might play an important role in the development and progression of diabetic nephropathy. Recent studies mainly focus on the effect of ApoE gene polymorphisms on the risk of diabetic nephropathy $(22,23)$. However, ApoE concentration significantly affects serum lipid levels, independent of ApoE polymorphism, by modulating lipoprotein production, lipolytic conversion and receptor-mediated clearance $(24,25)$. Therefore, studying the effect of ApoE polymorphisms on diabetic nephropathy without taking into account of ApoE concentrations may lead to confounding results. The previous studies on the association of ApoE level with diabetic nephropathy were limited and the study samples were small. One study found that subjects with type 1 diabetic nephropathy had an increased ApoE level (26). Another study used twodimensional electrophoresis to identify serum biomarkers for the diagnosis type 2 diabetic nephropathy and found that ApoE was a potential biomarker (27). The present study was the first large sample association study of lipids levels with type 2 diabetic nephropathy in Chinese, which included ApoE as one of the lipid metabolism parameters.

The present study also found that ApoE level was associated with ACR independent of other lipid parameters. Therefore, the mechanism of ApoE protein in diabetic nephropathy could not be merely attributed to ApoE-related lipid abnormalities. Some studies have shown that ApoE protein regulates mesangial cell proliferation and matrix production and it may act as an autocrine regulator of glomerular function $(28,29)$. Thus intrarenal dysfunction induced by an ApoE abnormality may contribute to the induction of diabetic nephropathy, however further studies are necessary to demonstrate this hypothesis. 
There are some limitations to our study. First, the crosssectional design of the present study was not sufficient to confirm a causal relationship between ApoE and albuminuria. It is well known that dyslipidemia can result from renal insufficiency. The underlying mechanisms included increased hepatic synthesis, decreased catabolism of lipoproteins and their delayed removal from plasma $(30,31)$. However, what was investigated in the present study was not advanced renal disease, and the mean eGFR of study subjects was $93.6 \mathrm{~mL} / \mathrm{min}$. Whether renal disease in the early stage could cause lipid abnormalities is still unknown. Second, we did not investigate the associations of ApoE gene polymorphisms with albuminuria. ApoE gene polymorphisms have been shown to be associated with serum ApoE level (32) and diabetic nephropathy in many studies $(22,23)$. The role of ApoE protein and ApoE gene polymorphisms in diabetic nephropathy could be examined simultaneously in the future. Third, the result of our study might not be generalizable due to the single-center design, and it should be examined in future multi-center studies.

In conclusion, the present study indicates that among the lipid parameters of total cholesterol, triglyceride, HDL-C, LDL-C, ApoA1, ApoB, ApoE and Lp(a), only the serum ApoE level was independently associated with urinary albumin excretion of type 2 diabetic patients from Shanghai, China.

The authors state that they have no Conflict of Interest (COI).

\section{Acknowledgement}

We are grateful to all subjects who participated in this study.

\section{References}

1. Coonrod BA, Ellis D, Becker DJ, et al. Predictors of microalbuminuria in individuals with IDDM. Pittsburgh Epidemiology of Diabetes Complications Study. Diabetes Care 16: 1376-1383, 1993.

2. Dominguez JH, Tang N, Xu W, et al. Studies of renal injury III: lipid-induced nephropathy in type II diabetes. Kidney Int 57: 92104, 2000.

3. Jenkins AJ, Lyons TJ, Zheng D, et al; DCCT/EDIC Research Group. Lipoproteins in the DCCT/EDIC cohort: associations with diabetic nephropathy. Kidney Int 64: 817-828, 2003.

4. Tolonen N, Forsblom C, Thorn L, et al. Lipid abnormalities predict progression of renal disease in patients with type 1 diabetes. Diabetologia 52: 2522-2530, 2009.

5. Hirano T, Sakaue T, Misaki A, et al. Very low-density lipoproteinapoprotein CI is increased in diabetic nephropathy: comparison with apoprotein CIII. Kidney Int 63: 2171-2177, 2003.

6. Ding G, van Goor H, Ricardo SD, Orlowski JM, Diamond JR. Oxidized LDL stimulates the expression of TGF-beta and fibronectin in human glomerular epithelial cells. Kidney Int 51: 147-154, 1997.

7. Nakhjavani M, Esteghamati A, Khalilzadeh O, Asgarani F, Mansournia N, Abbasi M. Association of macroalbuminuria with oxidized LDL and TGF-beta in type 2 diabetic patients: a casecontrol study. Int Urol Nephrol 42: 487-492, 2010.

8. Nieuwdorp M, Mooij HL, Kroon J, et al. Endothelial glycocalyx damage coincides with microalbuminuria in type 1 diabetes. Diabetes 55: 1127-1132, 2006.
9. Singh A, Satchell SC, Neal CR, McKenzie EA, Tooke JE, Mathieson PW. Glomerular endothelial glycocalyx constitutes a barrier to protein permeability. J Am Soc Nephrol 18: 2885-2893, 2007.

10. Abrass CK. Cellular lipid metabolism and the role of lipids in progressive renal disease. Am J Nephrol 24: 46-53, 2004.

11. Tseng $\mathrm{CH}$. Lipid abnormalities associated with urinary albumin excretion rate in Taiwanese type 2 diabetic patients. Kidney Int 67: 1547-1553, 2005.

12. Tseng $\mathrm{CH}$. Differential dyslipidemia associated with albuminuria in type 2 diabetic patients in Taiwan. Clin Biochem 42: 10191024, 2009.

13. Alberti KG, Zimmet PZ. Definition, diagnosis and classification of diabetes mellitus and its complications. Part 1: diagnosis and classification of diabetes mellitus provisional report of a WHO consultation. Diabet Med 15: 539-553, 1998.

14. Cockcroft DW, Gault MH. Prediction of creatinine clearance from serum creatinine. Nephron 16: 31-41, 1976.

15. Friedewald WT, Levy RI, Fredrickson DS. Estimation of the concentration of low-density lipoprotein cholesterol in plasma, without use of the preparative ultracentrifuge. Clin Chem 18: 499-502, 1972.

16. Mahley RW. Apolipoprotein E: cholesterol transport protein with expanding role in cell biology. Science 240: 622-630, 1988.

17. Eichner JE, Dunn ST, Perveen G, Thompson DM, Stewart KE, Stroehla BC. Apolipoprotein E polymorphism and cardiovascular disease: a HuGE review. Am J Epidemiol 155: 487-495, 2002.

18. Ashford JW. APOE genotype effects on Alzheimer's disease onset and epidemiology. J Mol Neurosci 23: 157-165, 2004.

19. Saito T, Matsunaga A, Oikawa S. Impact of lipoprotein glomerulopathy on the relationship between lipids and renal diseases. Am J Kidney Dis 47: 199-211, 2006.

20. Yorioka N, Nishida Y, Oda H, Watanabe T, Yamakido M. Apolipoprotein E polymorphism in $\operatorname{IgA}$ nephropathy. Nephron 83: 246249, 1999.

21. Asami T, Ciomartan T, Hayakawa H, Uchiyama M, Tomisawa $S$. Apolipoprotein E epsilon 4 allele and nephrotic glomerular diseases in children. Pediatr Nephrol 13: 233-236, 1999.

22. Mooyaart AL, Valk EJ, van Es LA, et al. Genetic associations in diabetic nephropathy: a meta-analysis. Diabetologia 54: 544-553, 2011.

23. Li Y, Tang K, Zhang Z, et al. Genetic diversity of the apolipoprotein $\mathrm{E}$ gene and diabetic nephropathy: a meta-analysis. Mol Biol Rep 38: 3243-3252, 2011.

24. Liberopoulos EN, Miltiadous GA, Cariolou M, Tselepis AD, Siamopoulos KC, Elisaf MS. The influence of serum apolipoprotein E concentration and polymorphism on serum lipid parameters in hemodialysis patients. Am J Kidney Dis 44: 300-308, 2004.

25. Haddy N, De Bacquer D, Chemaly MM, et al. The importance of plasma apolipoprotein $\mathrm{E}$ concentration in addition to its common polymorphism on inter-individual variation in lipid levels: results from Apo Europe. Eur J Hum Genet 10: 841-850, 2002.

26. Attman PO, Knight-Gibson C, Tavella M, Samuelsson O, Alaupovic P. The compositional abnormalities of lipoproteins in diabetic renal failure. Nephrol Dial Transplant 13: 2833-2841, 1998.

27. Kim HJ, Cho EH, Yoo JH, et al. Proteome analysis of serum from type 2 diabetics with nephropathy. J Proteome Res 6: 735-743, 2007.

28. Liberopoulos E, Siamopoulos K, Elisaf M. Apolipoprotein E and renal disease. Am J Kidney Dis 43: 223-233, 2004.

29. Chen G, Paka L, Kako Y, Singhal P, Duan W, Pillarisetti S. A protective role for kidney apolipoprotein E. Regulation of mesangial cell proliferation and matrix expansion. J Biol Chem 276: 4914249147, 2001.

30. Chan MK, Persaud JW, Ramdial L, Varghese Z, Sweny P, Moorhead JF. Hyperlipidaemia in untreated nephrotic syndrome, increased production or decreased removal? Clin Chim Acta 117: 
317-323, 1981.

31. Lee DM, Knight-Gibson C, Samuelsson O, Attman PO, Wang CS, Alaupovic P. Lipoprotein particle abnormalities and the impaired lipolysis in renal insufficiency. Kidney Int 61: 209-218, 2002.
32. Chasman DI, Kozlowski P, Zee RY, Kwiatkowski DJ, Ridker PM. Qualitative and quantitative effects of APOE genetic variation on plasma C-reactive protein, LDL-cholesterol, and apoE protein. Genes Immun 7: 211-219, 2006.

(C) 2011 The Japanese Society of Internal Medicine http://www.naika.or.jp/imindex.html 